\title{
An Overview of Peak-to-Average Power RATIO REDUCTION TECHNIQUES FOR OFDM SIGNALS
}

\author{
Zhuo Wang ${ }^{1}$, Enchang Sun ${ }^{2}$, Yanhua Zhang ${ }^{3}$ \\ ${ }^{1,2,3}$ College of Electronics Information and Control Engineering, Beijing University of \\ Technology, Beijing, China
}

\begin{abstract}
OFDM (Orthogonal Frequency Division Multiplexing) has been widely adopted for high data rate wireless communication systems due to its advantages such as extraordinary spectral efficiency, robustness to channel fading and better QoS (Quality of Service) performance for multiple users. However, some challenging issues are still unresolved in OFDM systems. One of the issues is the high PAPR (peak-toaverage power ratio), which results in nonlinearity in power amplifiers, and causes out of band radiation and in band distortion. This paper reviews some conventional PAPR reduction techniques and their modifications to achieve better PAPR performance. Advantages and disadvantages of each technique are discussed in detail. And comparisons between different techniques are also presented. Finally, this paper makes a prospect forecast about the direction for further researches in the area of PAPR reduction for OFDM signals.
\end{abstract}

\section{KEYWORDS}

OFDM, PAPR, Clipping, Companding

\section{INTRODUCTION}

OFDM (Orthogonal Frequency Division Multiplexing) [1]-[3] is a multicarrier orthogonal digital communication scheme where the whole available bandwidth is divided into many streams of low data rates and then modulated simultaneously by multiple carriers. It has been widely used for wireless applications such as Digital Audio Broadcasting (DAB), Terrestrial Digital Video Broadcasting (DVB-T), and IEEE 802.11a standard for Wireless Local Area Networks (WLAN) and IEEE 802.16a standard for Wireless Metropolitan Area Networks (WMAN). It provides greater immunity to multipath fading and impulse noise, and eliminates the need for equalizers, while efficient hardware implementation can be realized using Fast Fourier Transform (FFT) techniques.

When the several individual signals, which are modulated over a group of orthogonal subcarriers, are added up coherently, the problem of PAPR arises. PAPR is the ratio of the maximum power to the average power of a given signal. Whenever high PAPR occurs, the Digital-to-Analog Converter (DAC) and High Power Amplifier (HPA) require large dynamic ranges to avoid amplitude clipping, which increases both power consumption and component cost of the transceiver. Therefore, it is important and necessary to reduce the PAPR for making the system efficient. 
Various approaches have been proposed to reduce the PAPR such as clipping and filtering [4]-[8], peak windowing [9]-[11], companding [12]-[18], coding techniques [19]-[27], selected mapping [28]-[30], partial transmit sequence [31]-[35], interleaving [36][37], tone injection [38][39], tone reservation [38]-[40], active constellation extension [41] and so on. Although some techniques of PAPR reduction have been summarized in [42], it is still needed to give a comprehensive review including some motivations and innovations of PAPR reductions in recent years, such as reduced complexity and power saving. This paper also analyzes advantages and disadvantages of every techniques, and makes comparisons among them. In practical applications, different techniques should be chosen according to different systems. An effective technique should be given the better PAPR reduction performance and tradeoff among computational complexity, power consumption and Bit Error Rate (BER) performance, etc.

The rest of this paper is organized as follows: Section 2 gives a brief review of the definition of OFDM and PAPR. In Section 3, different PAPR reduction techniques are introduced and many modified methods based on these techniques, which have been proposed in recent years, are also presented. The criteria of PAPR reduction in OFDM systems is discussed in detail in Section 4, and then a summary of the lessons learned and suggestions on choosing proper PAPR reduction techniques in practical systems are provided in Section 5. Finally, we conclude this survey in Section 6.

\section{OfdM SySTEM MOdel AND PAPR}

In a typical OFDM system, frequency bandwidth $B$ is divided into $N$ non-overlapping orthogonal subcarriers of bandwidth, where $B=N \Delta f, \Delta f=1 / N T$. Then, each subcarrier of a given OFDM symbol is modulated by a known constellation. Let $X=\left[X_{0}, X_{1}, \cdots, X_{N-1}\right]^{\mathrm{T}}$ denote input symbol vector in the frequency domain, where $X_{n}$ represents the complex data of the $n^{\text {th }}$ subcarrier. Then after performing an $N$ length Inverse Fast Fourier Transform (IFFT) on $X$, we obtain time domain OFDM sequences $x=\left[x_{0}, x_{1}, \cdots, x_{N-1}\right]^{\mathrm{T}}$, where

$$
x(\mathrm{t})=\frac{1}{\sqrt{N}} \sum_{n=0}^{N-1} X_{n} e^{j 2 \pi n \Delta t}, 0 \leq t \leq N T .
$$

Due to the statistical independence of all subcarriers, the instantaneous amplitudes of the different signals may have high peaks aligned at the same time, leading to high PAPR which can be defined as:

$$
\text { PAPR }=\frac{\max _{n=0,1, \cdots, N-1}|x(t)|^{2}}{E\left[|x(t)|^{2}\right]}
$$

where $E[\cdot]$ denotes the expected operator. To better approximate the PAPR of OFDM signals, the OFDM signal samples are usually performed by $L$ times oversampling. So these $L$ times oversampled time domain samples are $L N$ point IFFT of the data block with $(L-1) N$ zero padding. Therefore, the PAPR that are computed from the $L$ times oversampled time domain signal samples is given by [42]

$$
\text { PAPR }=\frac{\max _{0 \leq k \leq N L-1}\left|x_{k}\right|^{2}}{E\left(\left|x_{k}\right|^{2}\right)}
$$

where $x_{k}$ are samples of the OFDM signal. 


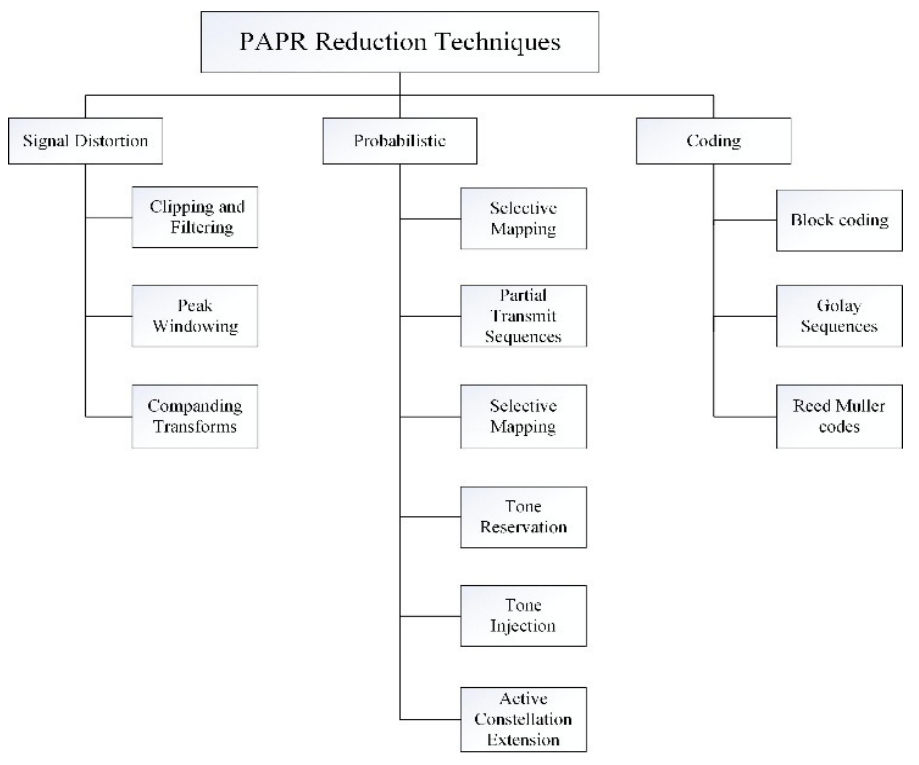

Figure 1. Classification of PAPR reduction techniques

\section{PAPR REDUCTION TECHNIQUES}

Numerous techniques have been proposed and optimized in the literature to reduce the PAPR of OFDM signals. These techniques are usually classified into three main categories: signal distortion techniques, probabilistic techniques and coding techniques as shown in Figure 1.

In this section, we will briefly review the principles of these PAPR reduction techniques. At the same time, we mainly focus on the important optimization among these techniques and point out the advantages and disadvantages of them.

\subsection{Signal Distortion Techniques}

Signal distortion techniques, including clipping and filtering, peak windowing and companding, distort the OFDM signals before power amplifier to reduce PAPR. These techniques are able to | reduce PAPR in great level, but result in signal distortion, which leads to worse BER_performance.

\subsubsection{Clipping and Filtering}

Amplitude clipping is a very basic and simple method to reduce PAPR. It employs a clipper that is used to limit the amplitude peaks of the input signal to a predetermined value if the signal exceeds it. Otherwise, the clipper passes the signal without any change. It could be described by a function as:

$$
B(x)= \begin{cases}\mathrm{x}, & |x| \leq A \\ A e^{j \phi(x)}, & |x|>A\end{cases}
$$

where $x$ is the original OFDM signal, $\phi(\mathrm{x})$ is the phase of $x, A$ is the predetermined clipping level, and $B(x)$ is the signal after clipping. Clipping method introduces in-band distortion and outof-band radiation, both due to its nonlinear processing, which can degrade the system performance such as BER and spectral efficiency. Filtering the clipped OFDM signal is a good method to reduce or remove out-of-band radiation, but it may cause some peak regrowth, because the signal 
after clipping and filtering may exceed the clipping level at some points. To solve this problem, Repeated Clipping and Filtering (RCF) [6] operation is generally used by clipping the oversampled time domain signals followed by filtering the clipped signals at the cost of additional computational complexity. Then a novel Iterative Clipping and Filtering (ICF) technique was proposed in [7], which could obtain a more desirable PAPR reduction level and cancel the distortion caused by clipping noise. But the major limitation of this method is requiring many iterations, resulting to high complexity.

Therefore, various methods proposed in some papers were developed to optimize ICF technique majoring in reducing computational complexity. Reference [43] presented a modified ICF scheme which limits the distortion on each subcarrier of the OFDM signals to achieve both low PAPR and low BER with fast convergence. In [44], the authors proposed an optimized ICF method which determines an optimal frequency response filter for each iteration using convex optimization to minimize signal distortion, so that the PAPR is reduced under a specified threshold. The authors claimed that this method achieves a desired PAPR reduction after only 1 or 2 iterations, whereas the conventional clipping and filtering method requires about 8 to 16 iterations to achieve the similar PAPR reduction. A novel Custom Conic Optimized Iterative Adaptive Clipping and Filtering (COIACF), which is also known as convex optimization, was proposed in [45] to reduce the PAPR of OFDM signals with lower complexity and bit error rate. The authors modified the filter response to produce a better reduction in PAPR and a better performance in in-band distortion and out-of-band radiation in less number of iterations due to the functionality of FFT.

\subsubsection{Peak Windowing}

This method reduces the peak value through multiplying a window function by the original OFDM signals [9]-[11], which can be used to suppress out-of-band radiation after clipping. The main idea of this method is that the valleys of window function are multiplied by the signal peaks while the high peaks of window function are multiplied by the signal valleys. Many window functions can be used in this process as long as they have good spectral properties. Hamming, Hanning and Kaiser windows are the most widely used window functions. This scheme attenuates signal peaks in a much smoother way, but the peak reduction worsens as the number of peak signals increases.

\subsubsection{Companding Transform}

Companding transform is one of the most attractive techniques of PAPR reduction with lower computational complexity that is not affected by the number of subcarriers. And side information is not necessary to be transmitted, leading to no loss in bit rates.

Companding transforms are firstly and typically applied to speech signals to optimize the required number of bits per sample. Since OFDM and speech signals have some similarities in the aspect that high peaks occur infrequently, same companding transforms can also be used to reduce the OFDM signal's PAPR [34].

In fact, the companding transforms can be viewed as an optimized clipping scheme. The biggest difference between them is that companding transforms enlarge the small signals while compressing the large signals in order to eliminate interference from noise, whereas clipping method does not change the small signals. This is the exact reason why clipping method suffers from in-band distortion and out-of-band radiation. Additionally, clipping method deliberately clips large signals when the amplitudes of the original OFDM signals are larger than the given threshold, resulting in clipped signals unrecoverable at the receiver. However companding transforms compand original OFDM signals using the strict monotone increasing function. So the companded 
signals at the transmitter can be recovered correctly and easily through the corresponding inversion of the transform function at the receiver.

Companding transform is an extra operation after the modulation of OFDM signals which definitely generates companding distortion. Hence, how to reduce the negative impact of companding distortion on the BER performance is the key part in designing companding transform. The first applicable nonlinear companding transform is the $\mu$-law companding that mainly focuses on preserving the high peaks and enhancing the low amplitudes of the signals. Therefore it keeps the peak power unchanged but increases the average power, leading to reduce of the PAPR [12]. The resulting companded signal $x_{\mathrm{c}}[n]$ using the $\mu$-law companding can be expressed as [13]:

$$
x_{c}[n]=\frac{A \operatorname{sgn}(x[n]) \log [1+\mu|x[n] / A|]}{\log (1+\mu)}
$$

where $A$ is a normalization constant such that $0 \leq|x[n] / A| \leq 1, \mu$ is the companding parameter and $\operatorname{sgn}(x[n])$ denotes the sign of $x[n]$. Figure 2 shows that the uncompanded envelope of an OFDM signal $x[n]$ and the $\mu$-law companded envelope corresponding to it with $\mu=3$.

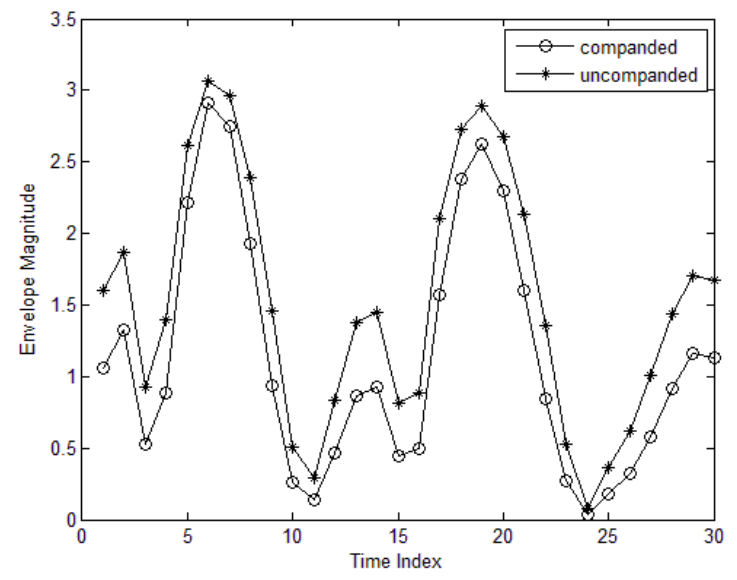

Figure 2. Uncompanded and companded using $\mu$-law OFDM envelopes

However, the $\mu$-law companding scheme reduces PAPR at the expense of the increase in the average signal power. Later, error companding transforms and exponential companding transforms were developed in [14] and [16], respectively. Both of them have less impacts on the original power spectrum comparing to the $\mu$-law companding scheme. It is the major reason that the error companding schemes and the exponential companding schemes not only enlarge the small amplitude signals but also compress the large amplitude signals. Both maintain the average power invariable by properly choosing parameters, which prevents small amplitude signals from noise. Reference [17] proposed a new companding scheme that transforms the Gaussian distributed signal into a distribution form with a linear piecewise function. Although, this companding scheme can reduce PAPR effectively, the computational complexity is increased at the same time. Then, a low-complexity linear companding transform (LCT) was investigated in [46] to reduce peak power by linearly transforming the small and large signal amplitudes with different scales. But this

| method can_not keep signal power at the same level for the input and output. Also, additional side information was needed at the decompanding operation, because LCT method does not have oneto-one mapping. In order to maintain the average signal power unchangeable and obtain a one-toone mapping, a new scheme was investigated in [47], called two-piecewise companding (TPWC) scheme, which transforms small amplitudes with a scale and large amplitudes with both a scale 
and a shift. A new piecewise linear companding transform was designed in [48], in which the signals with amplitudes over a given companded peak amplitude are clipped for peak power reduction, and the signals with amplitudes close to the given companded peak amplitude are linearly scaled for power compensation. The authors proved that this proposed piecewise linear companding scheme can reduce companding distortion and PAPR effectively. Besides, uniform companding[15], logarithmic companding and hyperbolic tangent companding transforms [18], with proper companding coefficients, were used in the literature to reduce PAPR.

\subsection{Probabilistic Techniques}

Due to the high PAPR appears randomly, these techniques focus on lower probability of high peaks of OFDM signals. The principle of these techniques is that different scrambling sequences are weighted to original OFDM signals to optimize phase sequences. Then, one combination between OFDM signal and phase sequence that has minimum PAPR is selected to be transmitted. The probability of high peaks occurrence will be reduced through this way and then PAPR is reduced.

\subsubsection{Selective Mapping}

Selective Mapping (SLM) is a relatively simple approach to reduce PAPR. A block diagram of the SLM technique is shown in Figure 3 [51]. The transmitter generates a set of sufficiently different candidate data blocks, all representing the same information as the original data blocks. Then each data block is multiplied by $U$ different phase sequences $B(u)=\left[b_{u, 0}, b_{u, 1}, \cdots, b_{u, \mathrm{n}-1}\right]^{T}, u=1,2, \cdots, U$. After combining phase factors with data blocks, the modified data blocks $X(u)=\left[X_{0} b_{u, 0}, X_{1,} b_{u, 1}, \cdots X_{N-1} b_{u, N-1}\right]^{T}, u=1,2, \cdots, U$ are obtained. Then IFFT is performed on the sub-blocks and the time domain signals become

$$
x^{(u)}(t)=\frac{1}{\sqrt{N}} \sum_{n=0}^{N-1} X_{n} b_{u, n} e^{j 2 \pi n \Delta f t}, 0 \leq t \leq N T, u=1,2, \cdots U .
$$

Among the modified data blocks $X(u)$, only one with the lowest PAPR is selected for transmission and the corresponding selected phase factor $b_{u, n}$ is also transmitted to receiver as side information to allow the recovery of original symbol sequences, which results in the data transmission rate loss. $\log _{2} u$ bits are needed as side information (SI) and $U$ times inverse discrete Fourier transform (IDFT) operations are also implemented in this process. So the extent of PAPR reduction achieved and the computational complexity for SLM depend on the number and design of the phase sequences.

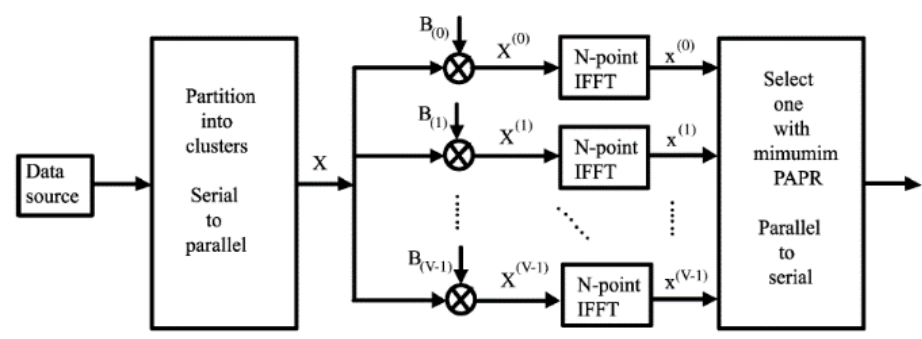

Figure 3. Block diagram of the SLM technique [51]

In the conventional SLM scheme, although, SI allows the recovery of original symbol sequences at the receiver, it will reduce the data transmission rate. On the other hand, an erroneous detection of the SI at the receiver may lead to a significant performance deterioration on the BER. Therefore, 
several blind SLM schemes [53]-[56] were produced to transmit signals without side information. In [55], the maximum likelihood decoder is firstly produced, which shows the same BER performance as the conventional SLM scheme assuming perfect side information recovery, but it causes large decoding complexity at the receiver. A new blind SLM scheme with low decoding complexity was then proposed in [56], in which the side information is embedded into each phase sequence by giving the phase offset to the elements of the phase sequences, which are determined by the bi-orthogonal vectors for the partitioned sub-blocks. At the same time, a maximum likelihood decoder with lower decoding complexity was derived for this scheme, which reduces the decoding complexity by $(M-2) / M$ compared with the conventional blind SLM scheme in [55], where $M$ denotes the times of IDFT operations. In [57], the authors proposed a suboptimal blind method based on Dummy Sequences Insertion (DSI) technique to reduce the PAPR level. Only a few sequences of zeros need to be inserted into the original signal. Besides, the signal recovery at the receiver is guaranteed by this embedded signaling, which is based on calculating the energy of the received signal. Any explicit SI is not needed to be transmitted in this proposed method, and simulations showed that this method does not only serve to reduce the PAPR level but also achieves a better BER performance.

Additionally, many attempts in the literature have been made to solve the problem of increased computational complexity, since the size of OFDM blocks may be large and the number of phase sequences $M$ and IFFT operations may be huge [58]-[61], when a substantial PAPR reduction is required. In order to address the problem of multiple IFFT operations, some new architectures have been proposed [60] [61], in which the frequency domain phase rotation operations are converted into time domain equivalent operations. However, the selections of sequences are limited and the adopted sequences are not randomly generated, resulting that the performance of PAPR reduction is affected. In view of the above literature, reference [62] proposed a new low complexity architecture, in which frequency domain cyclic shifting, complex conjugate and subcarrier reversal operations are all applied to increase the diversity of the candidate signals instead of only frequency domain phase rotation used in traditional SLM scheme. These four frequency domain operations are all converted into time domain equivalents similar to [60] [61] to avoid the problem of multiple IFFT operations. Then, the authors adopted various ways of partitioning and reassembling the subcarriers according to different phase rotations to reduce the computational complexity. The theoretical analysis and simulation results showed that this proposed scheme almost has the same PAPR reduction performance as the traditional SLM method but with a significantly reduced computational complexity.

\subsubsection{Partial Transmit Sequences}

In the PTS technique, an input data block containing $N$ symbols is partitioned into many disjoint sub-blocks, on which IFFT is performed separately and then weighted by a phase factor. The phase factors are selected in such a way as to minimize the PAPR of the combined signals of all the subblocks. Figure 4 [51] shows the block diagram of the PTS technique. Let an input data block $X=\left[X_{1}, X_{2}, \cdots, X_{N}\right]$ be partitioned into $M$ disjoint sub-blocks $X_{m}=\left[X_{m, 1}, X_{m, 2}, \cdots, X_{m, N}\right], 1 \leq m \leq M$, any two of these sub-blocks are orthogonal and $X$ is denoted as the combination of all sub-blocks $X=\sum_{m=1}^{M} X_{m}$. Then the IDFT operation is performed on each sub-block $X_{m}$, and weighted by a complex phase factor $b_{m}=e^{j \phi_{m}}$, where $\phi_{m}=[0,2 \pi), \mathrm{m}=1,2, \cdots, M$. The set of phase factors is denoted as a vector $b=\left[b_{1}, b_{2}, \cdots, b_{M}\right]^{T}$. The time domain signals after combining can be described as $x=\sum_{m=1}^{M} b_{m} \cdot x_{m}$, where $x_{m}$ is the time domain signal of $X_{m}$. Then the 
objective is to find the set of phase factors that minimizes the PAPR of $x$, which is chosen for transmission.

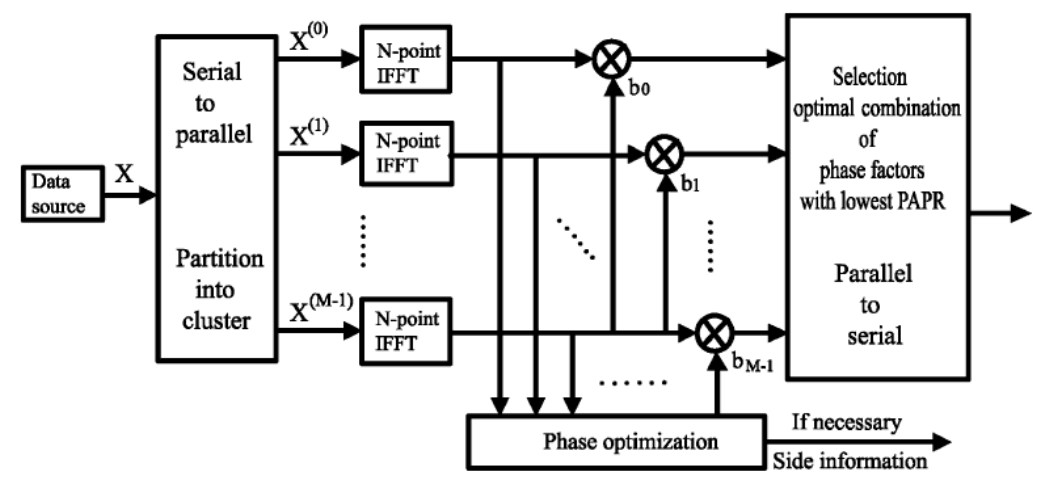

Figure 4. Block diagram of the PTS technique [51]

In general, the number of phase factors is limited to a set in order to reduce search complexity in the process of selecting the optimum one. The set of allowed phase factors can be described as $P=e^{\frac{e^{j 2 \pi k}}{W}}$, where $W$ is the number of allowed phase factors. The phase factor $b 1$ is usually set to 1 without any loss of performance, therefore, $M-1$ phase factors are alternative to be found and $W^{M-1}$ sets of phase factors are searched to find the optimum one. The performance of PAPR reduction and the search complexity depend on $M$ and $W$, since PTS needs $M$ IDFT operations for each data block, and the number of required side information bits is $\left\lfloor\log _{2} W^{M-1}\right\rfloor$, where $\lfloor y\rfloor$ denotes the smallest integer that does not exceed y [42]. Another factor that may affect the PAPR reduction performance in PTS is the method of division of sub-block partitioning. There are three kinds of sub-block partitioning schemes: adjacent, interleaved, and pseudo-random partitioning. Among them, pseudo-random partitioning has been proved to be the best choice, which can be seen from Figure 5, where the original data block is partitioned into four sub-blocks and QPSK is used in this simulation.

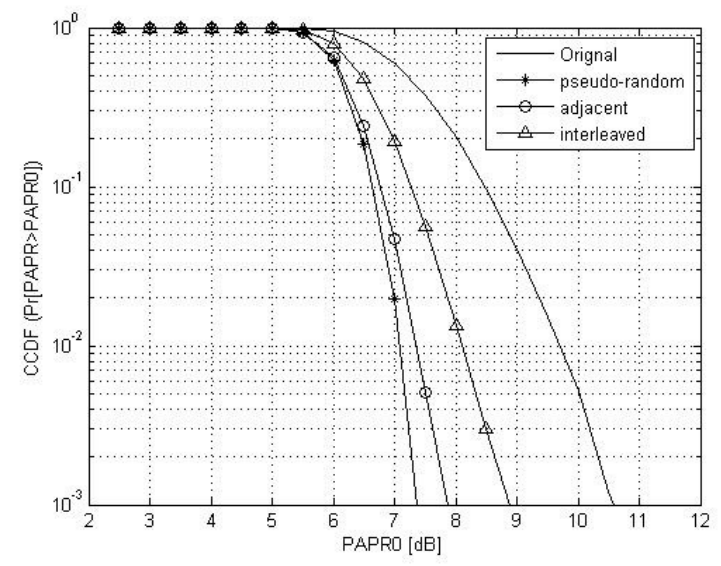

Figure 5. CCDF for sub-block partitioning schemes

How to reduce the high computational complexity for searching the optimal phase factors while maintaining a substantial reduction in PAPR has always been a huge problem. Various methods have been proposed in the literature to reduce the computational complexity in PTS, including iterative flipping algorithm [63], gradient descent search [64], sign-selection algorithms [65], 
genetic algorithm [66], sphere decoding algorithm [67], adaptive PTS approach [68] and many other optimized methods. Reference [69] proposed a modified flipping algorithm based on iterative flipping algorithm. This new technique partitions the formed sub-blocks into even and odd number of groups and then 'flipping' operation will be applied into group by group. That means two groups of four sub-blocks are formed after performing IDFT operations. Then the best combination between phase sequences and groups, which gives minimum PAPR value, is selected to be transmitted. Simulation result showed that this proposed flipping scheme offers better PAPR reduction and performance/complexity tradeoff than iterative flipping algorithm and conventional PTS. A novel PTS scheme using genetic algorithm with lower computational complexity was proposed in [70], in which candidate signals were generated using a set of matrices called seed matrices for a given weight set and block size. Through this way, one candidate sequence can be generated by combination of other sequences, since high degree of correlation is exhibited among them. Therefore, computational complexity is reduced without decreasing the number of subblocks and candidate signals.

\subsubsection{Interleaving}

The interleaving technique is extremely similar to SLM technique. In this method, a set of interleavers, devices that permute or reorder symbols in a specific way, instead of phase sequences is used to reduce the PAPR. So original data block $X=\left[X_{0}, X_{1}, \cdots, X_{N-1}\right]^{T}$ becomes many different permuted blocks by the use of these interleavers. Then the IDFT operation is performed on each one of these different permutations separately to generate multiple OFDM signals. The one with the smallest PAPR is chosen for transmission. To recover the original data block correctly, the receiver only need to know which interleaver is used at the transmitter, thus, the number of required side information bits is $\log _{2} k$, where $k$ represents the number of interleavers. So the amount of PAPR reduction and the degree of complexity in this method depend on $k$ and the design of the interleavers.

\subsubsection{Tone Reservation}

The basic idea of Tone Reservation (TR) is that a small number of subcarriers that are also called tones are not used for data transmission instead for PAPR reduction. A structured time domain vector $c$ is added to the original OFDM signal $x$ to change its statistical distribution to reduce PAPR. If we add a frequency domain vector $C=\left[C_{0}, C_{1}, \cdots, C_{N-1}\right]^{T}$ to $X, X$ and $C$ are restricted to lie in disjoint frequency subspaces, the new time domain signal can be represented as $x^{\prime}=x+c=I D F T\{X+C\}$. Then the objective is to find the proper time domain signal $c$ to be added to make the new time domain signal $x^{\prime}$ with low PAPR. Assume that there are $L$ unused tones in the OFDM signal, correspondingly a set of $L$ nonzero subcarriers in $C$, that is $X_{n}=0, n \in\left\{i_{1}, i_{2}, \cdots, i_{L}\right\}$ and $C_{n} \neq 0, n \in\left\{i_{1}, i_{2}, \cdots, i_{L}\right\}$. The $L$ nonzero positions in $C$ are called peak reduction carriers (PRCs). Since subcarriers of OFDM signals are orthogonal, these PRCs have no distortion effect on the data bearing subcarriers. To find the correct value of $C_{n}, n \in\left\{i_{1}, i_{2}, \cdots, i_{n}\right\}$, it could draw support from a convex optimization problem, which can be cast as a linear programming (LP) problem of complexity $o\left(L N^{2}\right)$ [38][78]. The receiver only needs to know the positions of these tones, which are served as overhead information.

Many different kinds of methods based on TR technique have been proposed in the literature. Clipping Control TR (CC-TR) [72], as the simplest TR scheme, in which the original signal is clipped firstly, then the signal above the predetermined threshold is mapped to the reserved carrier in frequency domain to reduce the PAPR. But some disadvantages lie in this method such that 
excessive iterations and slow convergence rate. The Adaptive Amplitude Clipping TR (AAC-TR) method and least squares approximation based TR (LSA-TR) method were introduced in [73] and [74] respectively. Both of them overcame shortcomings in CC-TR method, and had a better performance in PAPR reduction and computational complexity. In [75], authors proposed a new method based on the optimal prototype signal and least squares approximation algorithm, which were used to approximate the clipping noise just in the time domain by circularly shift and scale to reduce the PAPR of OFDM signals. In [76], an improved TR method based on the maximum likelihood estimate (MLE), called the MLE-TR algorithm, was proposed. This algorithm generates peak-canceling signals by use of the clipping noise that is created in clipping operation, and then uses the MLE algorithm to get an optimized factor to make the amplitude of peak canceling signals close to the clipping noise. Thus a better performance of PAPR reduction can be achieved in the case of a fast convergence. A novel curve fitting based tone reservation (CF-TR) method was investigated in [77], the key idea behind this method is to fit the peak-canceling signal waveform to the clipping noise waveform to get the peak canceling signal iterative. Besides, authors obtained the optimal clipping threshold that should be $A=\sigma \sqrt{\ln \frac{N}{M}}$, where $\sigma$ is the mean power of the OFDM signals, $M$ denotes peak reduction tones and $N$ is the number of data symbols. Simulation results showed that the proposed CF-TR method had better performance in PAPR reduction with computational complexity decreased.

\subsubsection{Tone Injection}

In this technique, the constellation size is increased in order to make each of the points in the original basic constellation mapped onto several other points in the expanded constellation. So the same information can be carried by any of these points, correspondingly some symbols of original OFDM signal are replaced by appropriate alternatives in an extended constellation, which is in favor of PAPR reduction. One point in the original basic constellation may be substituted for one in the expanded constellation, which is equivalent to injecting a tone of proper frequency and phase to the original OFDM signal. This is the origin of the name of this method. Assume that QAM is used as a modulation scheme and its original constellation size is $M$, the minimum distance between points in this square QAM constellation is $d$. The $k^{\text {th }}$ QAM symbol on a single subcarrier with all its original and expanded constellation points can be described as $X^{\prime}{ }_{k}=X_{k}+p_{k} D+j q_{k} D$, where $X_{k}$ is the $k^{\text {th }}$ original QAM symbol, $p_{k}$ and $q_{k}$ are integer numbers that are used to change the real and imaginary parts of $X_{k}$ respectively. $D$, a positive real number known at the receiver, represents the spacing between each original point in the original constellation and its equivalent points in the expanded constellation. The value of $D$ should be $D=\rho d \sqrt{M}$, where $\rho>1$, in order not to increase BER at the receiver [78]. The procedure of tone injection technique with 16-QAM constellation is shown in Figure 6 [79], where the original constellation point $A$ maps to one of $A_{i}, i=1,2, \cdots, 8$, according to changes in the value of $p_{k}$ and $q_{k}$. Then one of these expanded constellation points is chosen for transmission to reduce PAPR.

There is no loss of bit rates in tone injection technique because it does not need side information at all and only two simple modulo- $D$ operations are required at the receiver, which can be neglected in computational complexity. But tone injection technique can increase average signal power at the transmitter due to the use of enlarged signal constellation and the search complexity is also increased.

A new hexagonal constellation was proposed in [80]. More spaced signaling points can be packed using this hexagonal constellation than QAM constellation of the same area. So PAPR is reduced due to the extra degree of freedom and power increase is less compared to the QAM constellation. 
A new TI scheme that uses the clipping noise to find the optimal equivalent constellations is introduced in [81]. By projecting the clipping noise that is taken from the signals lager than a predetermined threshold to the nearest equivalent constellation points, the peak cancellation signal is easily determined, which reduces the complexity dramatically while maintaining a good PAPR performance. In [82] and [83], authors proposed a linear programming algorithm and parametric minimum method respectively as lower complexity solutions to the tone injection technique.

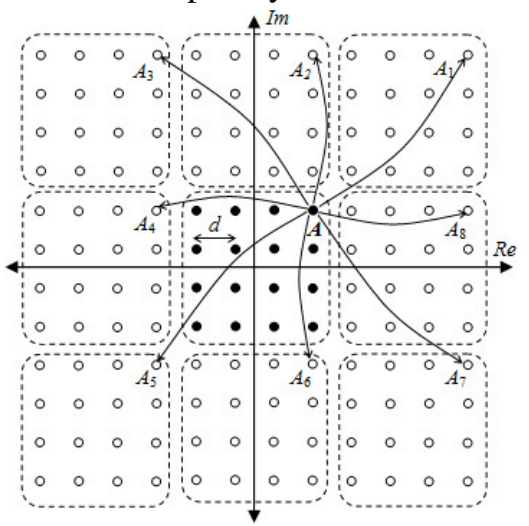

Figure 6 Tone injection technique for 16-QAM constellation [79]

\subsubsection{The Active Constellation Extension Technique}

The key idea behind the ACE method is to extend outer boundaries of the signal constellations in frequency domain. That is to say, some constellation points are extended toward the outside of the original constellation such that the PAPR of the OFDM data blocks in time domain may be reduced. The principles can be explained in Figure 7 [79] and Figure 8 [79], where QPSK and 16QAM modulation assumed to be used. The shaded regions in Figure 7 and Figure 8 and the straight lines starting at the non-corner constellation points to infinity in Figure 8 represent the region of increased margin for the data symbols. A reasonable combination of these additional signals can be partly used to cancel the peaks of time domain signals and then reduce the PAPR of the transmit signals. Waveforms of original signals and after ACE and the constellations are shown in Figure 9 and Figure 10 respectively with QPSK modulation and 256 subcarriers.

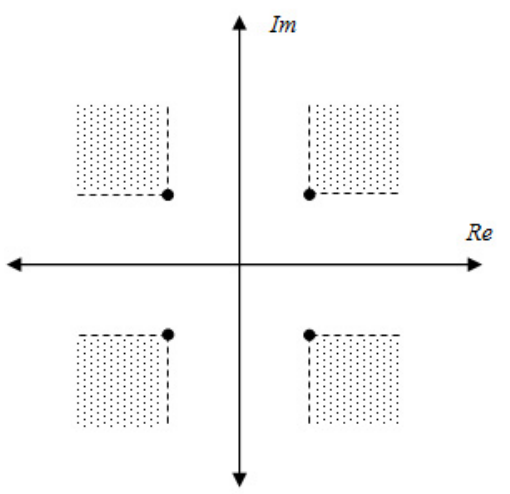

Figure 7 Active constellation extension technique with QPSK modulation [79]

Comparing Figure 9 and Figure 10, it is shown that the ACE method is more applicable to small constellations, because all the points in the QPSK constellation can be extended, nevertheless, only small parts of the points in the 16-QAM constellation have room for increased margin. In addition, there is no loss in data rates and no side information is required at the receiver. Furthermore, the increase in average transmit power resulted from constellation extention can be neglected, which 
leads to almost no significant degradation in BER performance. For practical implementation, fast convergence rate of ACE algorithm is an important issue. Smart gradient-project (SGP) algorithm [41], adaptive scaling (AS) algorithm [84] and adaptive clipping control ACE algorithm [85] were introduced respectively for fast convergence rate. A modified method of adaptive clipping control ACE algorithm was proposed in [86]. By increasing the step size both for peak canceling signal and clipping level control, the convergence rate was improved with lower PAPR.

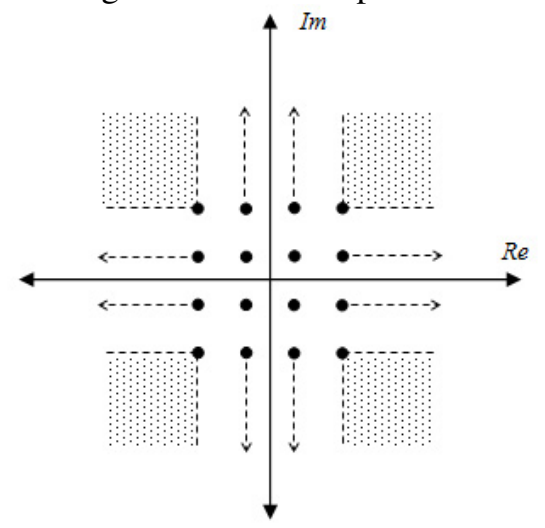

Figure 8 Active constellation extension technique with 16-QAM modulation [79]
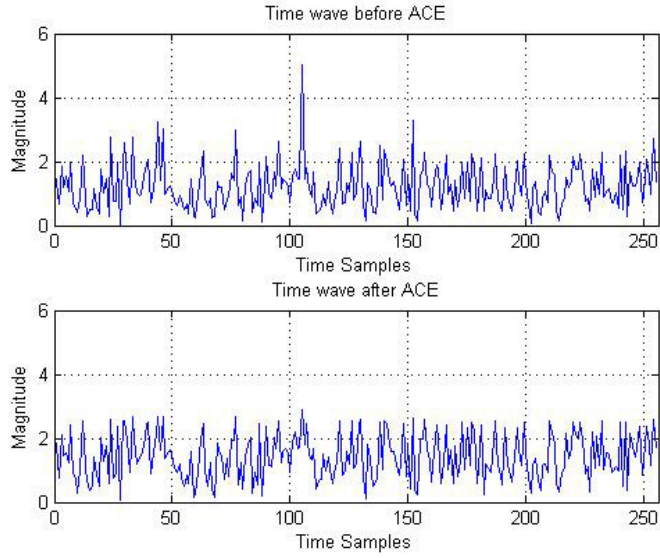

Figure 9 Waveforms before ACE and after ACE

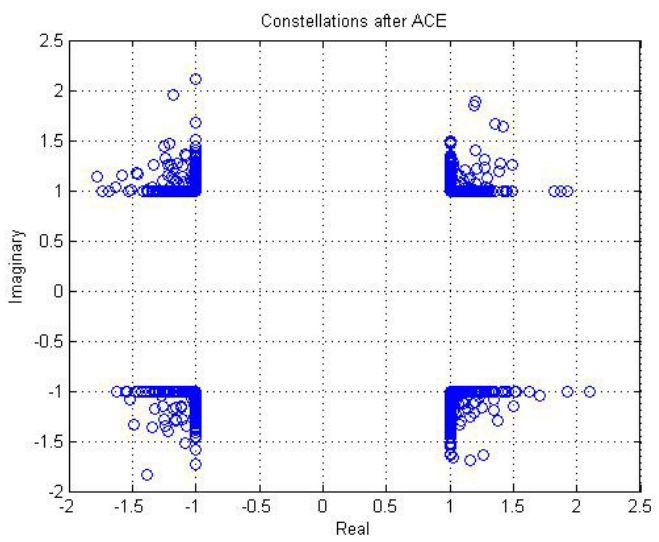

Figure 10 Constellations after ACE with QPSK modulation 


\subsection{Coding Techniques}

The basic idea of coding techniques is to choose a set of code words with lower PAPR. Then the input data blocks are mapped into these selected code words by various coding method. Therefore some peak amplitudes that lie in small part of original OFDM signals may be disappeared such that PAPR is reduced.

A simple linear block coding (LBC) scheme was proposed in [19], where 3 bits are mapped into 4 bits code word by adding a parity bit. Then Cyclic Coding (CC) that is applicable for any number of subcarriers was introduced in [20], which can reduce PAPR by more than $3 \mathrm{~dB}$. In [88], Subblock Coding (SBC) scheme was proposed, where long data blocks are divided into many subblocks, each of them adds an odd parity bit. The position of the added parity bit has an influence on the degree of reduction of PAPR. All the above schemes require side information to be transmitted to the receiver, which need to know the locations of the parity bits. In [89], authors proposed a new scheme, in which standard arrays of linear block codes are used for PAPR reduction. The coset leaders of a linear code are used for scrambling, which leads to no side information required to be transmitted, and the received signals can be decoded by syndrome decoding at the receiver. A low complexity Complement Block Coding (CBC) scheme was proposed in [90], few complement bits are added to the original information bits to form a new code word to reduce the probability of the peak signals occurrence. $\mathrm{CBC}$ scheme has no restriction on frame size and has lower implementation complexity, therefore it is used more widely.

In [23], authors proposed to apply fountain codes to control the PAPR of OFDM signals at a desired level. The principle of this scheme is that original source packets are fountain encoded to a semi-infinite packet stream. Then the fountain coded packets are fed to a $N$ point IDFT operation and PAPR control block. The PAPR control block is used to check whether the PAPR of the output signal after IDFT operation is at the desirable level or not. If the PAPR level exceeds the predetermined threshold, this OFDM symbol will be discarded. Otherwise, this symbol will pass without any change. Once the receiver gets enough packets for recovering the original source packets successfully, the fountain encoder will stops the ongoing packet transmission. This scheme works well in practice since the codes are rateless, though the complexity may be little higher.

Golay complementary sequences with different constellations can be used as code words to achieve the PAPR reduction [91]-[93], more than $3 \mathrm{~dB}$ PAPR reduction can be obtained in this method. Reed Muller codes [94] is another efficient coding scheme. It separates the code words with high PAPR by dividing non-binary Reed-Muller codes into many cosets, thus PAPR is reduced.

Coding technique is a nonlinear processing procedure, which do not appear clipping noise like the clipping method. But it increases the complexity both at the transmitter and the receiver, due to the additional information added to the original data blocks, which also results in some data rate loss and bandwidth expansion.

Table1 Comparison between PAPR reduction techniques

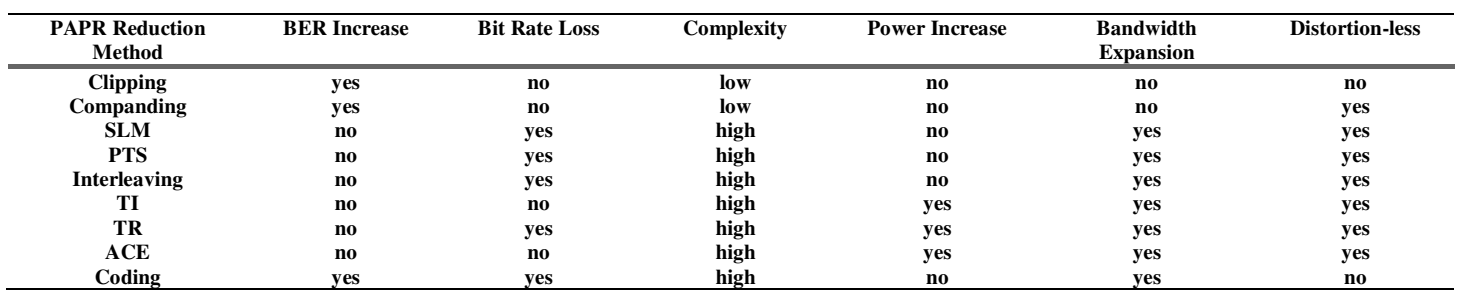




\section{Criteria for Selection of PAPR Reduction Techniques}

As mentioned above, every technique has their own advantages and disadvantages. So it is supposed to choose techniques or hybrid techniques (in which two or more techniques are combined) according to practical demand and some following factors should be considered before choosing the proper techniques.

High capability of PAPR reduction: Obviously, this is the primary and most important factor to be considered in selecting the PAPR reduction techniques. The level of PAPR reduction should be as large as possible when other factors are not greatly affected.

Low average power: The increase in average power can reduce PAPR, but it requires a larger linear operation region in HPA, which results in higher distortions and BER performance degradation.

Low computational complexity: Generally, the more complex the technique is, the more PAPR reduction capability it will get. However, complex techniques require additional time, hardware and power that should be as minimal as possible in overall systems.

Less bandwidth expansion: The bandwidth expansion occurs when side information is needed to be transmitted in some PAPR reduction techniques such as PTS and interleaving. Considering the bandwidth is a rare resource in communication systems, it is desirable to reduce PAPR at the expense of no bandwidth expansion.

Less BER performance degradation: BER performance degradation is also an important factor in communication system that should be considered. The goal in PAPR reduction is to get better system performance including BER.

Other Factors: Some nonlinear devices such as digital-to-analog converter, HPA and mixer also should be taken into consideration as they can protect PAPR reduction without nonlinear distortion. Additionally, the cost of these devices is also an important factor that should be considered in practical systems.

Table 1 summarizes some PAPR reduction techniques and performance impacting factors mentioned above. It can be clearly seen that no specific PAPR reduction technique achieves the best performance in all situations. Therefore, proper techniques should be selected based on practical systems, i.e. available resources and performance constraints.

\section{LESSONS LEARNED AND SUGGESTIONS}

The topic of PAPR reduction in OFDM systems is still an active area for further researches, since no PAPR reduction technique has been proved to be the best one. In practical application, global consideration should be taken into to help us to select proper techniques that can reduce PAPR largely and keep good performance in terms of the performance factors mentioned above at the same time.

In modern OFDM based wireless communication standards, high data rates and mobility are basic requirements, which need a large number of subcarriers to support them. For example, WiMAX, LTE and DVB-T support up to 512, 2048 and 8192 subcarriers respectively. A high number of subcarriers will lead to high PAPR but causes a huge problem in PAPR reduction: computational complexity. Probabilistic techniques are suffered from higher complexity due to the increase of the number of subcarriers. Take SLM and PTS methods for example, the number of subcarriers is denoted as $N, M$ represents the number of IFFT blocks as well as the number of phase sequences. For SLM technique, $3 M N\left(1+\log _{2} N\right)+M(N-1)-1$ additions and $2 M N\left(1+\log _{2} N\right)+M$ multiplications are needed, while $3 M N \log _{2} N+M[2 N(M+1)-1]$ additions and $2 M N \log _{2} N+2 N+1$ 
multiplications are needed in PTS method [79]. Clearly, it is shown that computational complexity grows rapidly as the number of subcarriers increases. Additionally, most of coding schemes are also limited to use in practical application due to their high implementation complexity and many of them are not applied to large number of subcarriers. Therefore, repeated clipping and filtering method with lower complexity is widely used in practical systems, under the condition of a tolerable BER. Many modifications in the literature have been proposed recently to solve the problem of high complexity such as blind SLM, genetic algorithm and iterative flipping algorithm in PTS, which are mentioned above. These attempts may lead to viability in practical systems, which seems to become a promising direction for further researches in the area of PAPR reduction for OFDM based systems.

Another issue that needs to be mainly focused on should be the transmitted power constraint, which means that the average power of transmitted signals is likely to be unchanged. Because the power amplifier's gain must be dynamically adjusted according to the power of the signal, which increases the hardware cost. Absolutely, it is desirable to maintain the average power constant with minimum extra complexity. Some PAPR reduction techniques including tone reservation, tone injection and ACE reduce PAPR at the cost of increasing the average power, while clipping and filtering method is an example that reduces average power. In addition, the concept of energy efficiency and green networks has been proposed and attracted increasing attention recently. Due to the fact that energy consumption of networks is growing fast and the trend of achieving green power in wireless communication systems is becoming popular, therefore, reducing power consumption and improving energy efficiency in power amplifier as much as possible at the process of reducing PAPR is a rich subject for conducting further research.

In future development of PAPR reduction techniques, practical potential should be firstly concerned rather than just seeking possible theoretical solutions. Therefore, an efficient PAPR reduction method in practical system should be the lower value of PAPR while keeping the minimum computational complexity and power consumption. This makes sense since other undesirable effects resulting from PAPR reduction techniques could be mitigated by other modules or the disadvantages could be acceptable in wireless systems as well as some performance metrics such as throughput, QoS and reliability are above a desirable level.

\section{Conclusions}

OFDM is an efficient modulation technique for high data rate transmission in wireless communication systems. However, high PAPR is one of the serious drawbacks of OFDM signals which may result in nonlinear distortions and spectral spreading in power amplifier. In this paper, we provides an overview of some conventional PAPR reduction schemes. All of them have the potential to provide substantial reduction in PAPR at the cost of data rate loss, increase in computational complexity, BER and transmit power and so on. Therefore, many modifications based on these conventional PAPR reduction schemes in the literature that are committed to performing better PAPR reduction level, reducing complexity, BER and transmit signal power are also summarized in this paper. Then comparisons among these techniques are presented and discussed. It is clear that any specific PAPR reduction technique has not been proved to be the best one so far, as each of them has its own advantages and disadvantages. Therefore, performance impacting factors that are mentioned above should be carefully chosen according to specific system requirements. Since high data rates need a large number of subcarriers and energy efficiency is the future trends in OFDM systems, we demonstrate that reducing computational complexity, due to the high number of subcarriers, and power consumption in power amplifier at the process of PAPR reduction is a promising direction for conducting further research in most OFDM based systems. 


\section{ACKNOWLEDGMENTS}

This work was financially supported by the National Natural Science Foundation of China under Grant 61372089 and 61571021, the Science and Technology Development Project of Beijing Municipal Education Commission under Grant KM201610005007.

\section{REFERENCES}

[1] Wu Y \& Zou W Y, (1995) "Orthogonal frequency division multiplexing: a multi-carrier modulation scheme”. IEEE Trans Consumer Electronics, Vol. 41, No. 3, pp 392-399.

[2] Zou W Y \& Wu Y, (1995) “COFDM: An overview”, IEEE Trans Broadcasting, Vol. 41, No. 1, pp 1-8.

[3] Hwang T, Yang C, Wu G, Li S, et al, (2009) “OFDM and its wireless applications: A survey”, IEEE Transactions on Vehicular Technology, Vol. 58, No. 4, pp 1673-1694.

[4] Panta K R \& Armstrong J, (2004) "Effect of clipping on the error performance of OFDM in frequency selective fading channels", IEEE Trans. Wireless Communications, Vol. 3, No. 2, pp 668-671.

[5] Ochiai H \& Imai H, (2000) "On the clipping for peak power reduction of OFDM signals", In: Proc. IEEE Global Communications Conference (GLOBECOM), San Francisco, USA, pp 731-735.

[6] Armstrong J, (2002) "Peak-to-average power reduction for OFDM by repeated clipping and frequency domain filtering”, IEEE Electronic Letters, Vol. 38, No. 2, pp 246-247.

[7] Chen H \& Haimovich. (2003) "A. An iterative method to restore the performance of clipped and filtered OFDM signals”, IEEE International Conference of Communications (ICC), Alaska,USA, pp 3438-3442.

[8] Kubo A, Tomisato S \& Hata M, (2005) "Transmission performance of highly efficient OFDM signals with iterative peak reduction", Proc. Asia-Pacific Conference on Wearable Computing Systems (APWCS), Sapporo, Japan, pp 145-148.

[9] Nee R V \& Wild A D, (1998) "Reducing the peak to average power ratio of OFDM", Proc. IEEE VTC, pp 18-21.

[10] Kim D, Shi D, Park Y, et al, (2005) "New peak-windowing for PAPR reduction of OFDM systems", Proc. Asia-Pacific Conference on Wearable Computing Systems (APWCS), pp 169-173.

[11] Cha S, Park M, Lee S, et al, (2008) "A new PAPR reduction technique for OFDM systems using advanced peak windowing method", IEEE Transactions on Consumer Electronics, Vol. 54, No. 2, pp 405-410.

[12] Pratt T G, Jones N, Smee L, et al, (2006) "OFDM link performance with companding for PAPR reduction in the presence of nonlinear amplification”, IEEE Trans. Broadcast, Vol. 52, No. 2, pp 261267.

[13] Wang X, Tjhung T T \& Ng C S (1999) "Reduction of peak-to-average power ratio of OFDM system using a companding technique”, IEEE Trans. Broadcast, Vol. 45, No. 3, pp 303-307.

[14] Jiang T \& Zhu G, (2004) "Nonlinear companding transform for reducing peak-to-average power ratio of OFDM signals”, IEEE Trans. Broadcast, Vol. 50, No. 3, pp 342-346.

[15] Jiang T, Xiang W, Richardson P C, et al, (2007) "On the nonlinear companding transform for reduction in PAPR of MCM signals”, IEEE Trans. Wireless Commun., Vol. 6, No. 6, pp 2017-2021.

[16] Jiang T, Yang Y \& Song Y, (2005) "Exponential companding transform for PAPR reduction in OFDM systems", IEEE Trans. Broadcast., Vol. 51, No. 2, pp 244-248.

[17] Wang Y, Ge J, Wang L, et al, (2013) "Nonlinear companding transform for reduction of peak-toaverage power ratio in OFDM systems”, IEEE Trans. Broadcast, Vol. 59, No. 5, pp 369-375.

[18] Lowe D \& Huang X, (2007) "Optimal adaptive hyperbolic companding for OFDM", Proc. 2nd International Conference on Wireless Broadband and Ultra Wideband Communications, Sydney, pp24-24.

[19] Jones A E, Wilkinson T A \& Barton S K, (1994), "Block coding scheme for reduction of peak to mean envelope power ratio of multicarrier transmission scheme", IEE Electronic Letters, Vol. 30, No. 22, pp 369-375.

[20] Wulich D, (1966), "Reduction of peak to mean ratio of multicarrier modulation using cyclic coding", IEE Electronic Letters, Vol. 32, No. 29, pp 432-433. 
[21] Fragicomo S, Matrakidis C \& OReilly J J, (1998) "Multicarrier transmission peak-to-average power reduction using simple block code", IEEE lectronic Letters, Vol. 34, No.14, pp 953-954.

[22] Hao M J \& Lai C H, (2010) "Precoding for PAPR reduction of OFDM signals with minimum error probability", IEEE Trans. Broadcast, Vol. 56, No. 1, pp 120-128.

[23] Jiang T \& Li X, (2010) "Using fountain codes to control the peak-to-average power ratio of OFDM signals", IEEE Trans. Veh. Technol, Vol. 59, No. 8, pp 3779-3758.

[24] Shokrollahi A, (2006) "Raptor codes", IEEE Trans. Inf. Theory, Vol. 52, No. 6, pp 2551-2567.

[25] Patterson K, (2000) "Generalized reed-muller codes and power control in OFDM modulation", IEEE Trans. Inf. Theory, Vol. 46, No. 1, pp 104-120.

[26] Schmidt K U, (2008) "On the peak-to-mean envelope power ratio of phaseshifted binary codes", IEEE Trans. Commun., Vol. 56, No. 11, pp 1816-1823.

[27] Sabbaghian M, Kwak Y \& Tarokh V, (2011) "New codes from dual BCH codes with applications in low PAPR OFDM”, IEEE Trans. Wireless Commun., Vol. 10, No. 12, pp 3990-3994.

[28] Goff S Y, Al-Samahi S S, Khoo B K, et al, (2009) "Selected mapping without side information for PAPR reduction in OFDM", IEEE Trans. Wireless Commun., Vol. 8, No. 1, pp 3320-3325.

[29] Joo H S, Heo S J, Jeon H B, et al, (2009) "A new blind SLM scheme with low complexity of OFDM signals”, Proc. IEEE Vehicular Technology Conference (VTC), Anchorage, AK, pp 1-5.

[30] Li C P, Wang S H \& Wang C L, (2010) "Novel low-complexity SLM schemes for PAPR reduction in OFDM systems”, IEEE Trans. Signal Process, Vol. 58, No. 5, pp 2916-2921.

[31] Jayalath A D S \& Tellambura C, (2000) "An adaptive PTS approach for the reduction of peak-toaverage power ratio of an OFDM signal", IEEE lectronic Letters, Vol. 36, No. 14, pp 1226-1228.

[32] Hou J, Ge J \& Li J, (2001) "Peak-to-average power ratio reduction of OFDM signals using PTS scheme with low computational complexity", IEEE Trans. Broadcast, Vol. 57, No. 1, pp 143-148.

[33] Taspinar N, Kalinli A \& Yildirim M, (2011) "Partial transmit sequences for PAPR reduction using parallel tabu search algorithm in OFDM systems", IEEE Commun. Lett., Vol. 15, No. 9, pp 974-976.

[34] Cho Y J, No J S \& Shin D J, (2012) "A new low-complexity PTS scheme based on successive local search using sequences", IEEE Commun.Lett, Vol. 16, No. 9, pp 1470-1473.

[35] Qi X, Li Y \& Huang H, (2012) "A low complexity PTS scheme based on tree for PAPR reduction. IEEE Commun. Lett.”, Vol. 16, No. 9, pp 1486-1488.

[36] Jayalath A D \& Tellambura C, (2000) "Reducing the peak to-average power ratio of orthogonal frequency division multiplexing signal through bit or symbol interleaving", IEE Electronic Letters, Vol. 36, No. 13, pp 1161-1163.

[37] Hill G R, Faulkner M \& Singh J, (2000) "Reducing the peak-to-average power ratio in OFDM by cyclically shifting partial transmit sequences”, Elect. Lett., Vol. 36, No. 6, pp560-561.

[38] Tellado J \% Cioffi J M, (2000) Peak to average power reduction for multicarrier modulation: [Ph.D. Dissertation]. Stanford: Information Systems Laboratory in Stanford University.

[39] Guan L \& Zhu A, (2011) Gaussian pulse-based two-threshold parallel scaling tone reservation for PAPR reduction of OFDM signals. International Journal of Digital Multimedia Broadcasting, Article ID 470310.

[40] Jiao Y Z, Liu X J \& Wang X A, (2008) “A novel tone reservation scheme with fast convergence for PAPR reduction in OFDM systems", Proc. IEEE 5th Consumer Communications and Networking Conference (CCNC), Las Vegas, USA, pp 398-402.

[41] Krongold B S \& Jones D L, (2003) "PAPR Reduction in OFDM via active constellation extension", IEEE Trans. Broadcast, Vol. 49, No. 3, pp 258-268.

[42] Han S H \& Lee J H, (2005) "An overview of peak-to-average power ratio reduction techniques for multicarrier transmission”, IEEE Personal Communications, Vol. 12, No. 2, pp 56-65.

[43] Deng S K \& Lin M C, (2007) "Recursive clipping and filtering with bounded distortion for PAPR reduction", IEEE Trans. Communications, Vol. 5, No. 1, pp 227-230.

[44] Wang Y C, \& Luo Z. Q, (2011) "Optimized iterative clipping and filtering for PAPR reduction of OFDM signals”, IEEE Trans. Communications, Vol. 59, No. 1, pp 33-37.

[45] Nandalal V \& Sophia S, (2014) "PAPR Reduction of OFDM Signal via custom conic optimized iterative adaptive clipping and filtering”, Wireless Pers. Communications, Vol. 78, No. 2, pp 867-880.

[46] Aburakhia S A, Badran E F \& Mohamed D A E, (2009) "Linear companding transform for the reduction of peak-to-average power ratio of OFDM signals”, IEEE Trans. Broadcast., Vol. 55, No. 1, pp 155-160.

[47] Yang P \& Hu A, (2011) "Two-piecewise companding transform for PAPR reduction of OFDM signals”, Proc. Wireless Commun. Mobile Comput. Conf. (IWCMC), Istanbul, Turkey, pp 619-623. 
[48] Hu M, Li Y, Wang W, et al, (2014) "A piecewise linear companding transform for PAPR reduction of OFDM signals with companding distortion mitigation”, IEEE Transactions on Broadcasting, Vol. 60, No. 3, pp 532-539.

[49] Gong H, Ye W, Feng S, et al, (2005) “A threshold companding scheme for reducing peak-to-average power ratio of OFDM signals", Proc. International Conference on Wireless Communications, Networking and Mobile Computing, Wuhan, China, pp 573-576.

[50] Yang G, Zhou Y \& Qian S, (2007) "Using hyperbolic tangent sigmoid transfer function for companding transform in OFDM systems". Proc. International Symposium on Communications and Information Technology, Sydney, pp. 87-90.

[51] Jiang T \& Wu Y, (2008) "An overview: peak-to-average power ratio reduction techniques for OFDM signals”, IEEE Transactions on Broadcasting, Vol. 54, No. 2, pp 257-268.

[52] Jeng S S \& Chen J M, (2011), "Efficient PAPR reduction in OFDM systems based on a companding technique with trapezium distribution", IEEE Trans. Broadcast., Vol. 57, No. 2, pp 291-298.

[53] Breiling H, Muller-Weinfurtner S H \& Huber J B, (2001) "SLM peak power reduction without explicit side information", IEEE Commun.Lett., Vol. 5, No. 6, pp 239-241.

[54] Baxley R J \& Zhou G T, (2005) "Map metric for blind phase sequence detection in selected mapping", IEEE Trans. Broadcast, Vol. 51, No. 4, pp 565-570.

[55] Jayalath A D S \& Tellambura C, (2005) "SLM and PTS peak-power reduction of OFDM signals without side information”, IEEE Trans. Wireless Commun., Vol. 4, No. 5, pp 2006-2013.

[56] Joo H-S, Heo S-J, Jeon H-B, et al, (2012) "A new blind SLM scheme with low decoding complexity for OFDM systems”, IEEE Trans. Broadcast., Vol. 58, No. 4, pp 669-676.

[57] Sghaier M, Abdelkefi F \& Siala M, (2014) "An efficient blind dummy zeros insertion and SLM scheme for PAPR reduction in OFDM systems", Wireless Communications and Networking Conference (WCNC), Istanbul, Turkey, pp 747-752.

[58] Park J, Hong E \& Har D, (2011) "Low complexity data decoding for SLM-based OFDM systems without side information”, IEEE Commun. Lett., Vol. 15, No. 6, pp 611-613.

[59] Jeon H B, No J S \& Shin D J, (2011) "A low-complexity SLM scheme using additive mapping sequences for PAPR reduction of OFDM signals” IEEE Trans. Broadcast., Vol.57, No. 4, pp 866-875.

[60] Wang C L \& Ouyang Y, (2005) "Low-complexity selected mapping schemes for peak-to-average power ratio reduction in OFDM systems”, IEEE Trans. Signal Process., Vol. 53, No. 12, pp 4652-4660.

[61] Wang S H, Sie J C \& Li P C, (2011) “A low-complexity PAPR reduction scheme for OFDMA uplink systems”, IEEE Trans. Wireless Commun., Vol. 10, No. 4, pp 1242-1251.

[62] Wang S H, Lee K C \& Li C P, (2015) "A low-complexity architecture for PAPR reduction in OFDM systems with near-optimal performance", IEEE Trans. Vehicular Technology, DOI: 10.1109/TVT.2015.2395818.

[63] Cimini L J \& Sollenberger N R, (2000) "Peak-to-average power ratio reduction of an OFDM signal using partial transmit sequences”, IEEE Commun. Lett., Vol. 4, No.3, pp 86-88.

[64] Han S H \& Lee J H, (2004) "PAPR reduction of OFDM signals using a reduced complexity PTS technique”, IEEE Signal Process. Lett., Vol. 11, No. 11, pp 887-890.

[65] Chen J C \& Wen C K, (2010) "A low-complexity scheme to reduce the PAPR of an OFDM signal using sign-selection algorithms”, IEEE Signal Process. Lett., Vol. 17, No. 2, pp 189-192.

[66] Kim S S, Kim M J \& Gulliver T A, (2006) A mew PTS for PAPR reduction by local search in GA. In: Proceedings of International Joint Conference on Neural Networks, Canada, pp 2370-2373.

[67] Alavi A, Tellambura C \& Fair I, (2005) "PAPR reduction of OFDM signals using partial transmit sequence: an optimal approach using sphere decoding”, IEEE Commun. Lett., Vol. 9, No.11, pp 982984.

[68] Hong E \& Har D, (2010) "Peak-to-average power ratio reduction in OFDM systems using all-pass filters", IEEE Trans. Broadcast., Vol. 56, No. 1, pp 114-119.

[69] Mangal S \& Kumar P S, (2015) On the performance analysis of partial transmit sequence using modified flipping algorithm for PAPR reduction in OFDM systems, omputer Communication Control and Information and Technology (C3IT) Third International Conference, Hooghly, pp 1-4.

[70] Shukla J, Joshi A, Bansal R, et al, (2014) PAPR reduction of OFDM systems using PTS with genetic algorithm at low computational complexity, IEEE International Conference on Recent Advances and Innovations in Engineering, Jaipur, India, pp 9-11.

[71] Jayalath A D S \& Tellambura C, (2000) The use of interleaving to reduce the peak-to-average power ratio of an OFDM signal. IEEE Globecom2000, San Francisco, USA, pp 82-86. 
[72] Gatherer A \& Polley M, (1997) Controlling clipping probability in DMT transmission. Proc. 31st Asilomar Conference on Signals, Systems, and Computers., CA, USA,pp 578-584

[73] Wang Y, Chen W \& Tellambura C, (2012) "Genetic algorithm based nearly optimal peak reduction tone set selection for adaptive amplitude clipping PAPR reduction”, IEEE Trans. Broadcast., Vol. 58, No. 3, pp 462-471.

[74] Li H, Jiang T \& Zhou Y, (2011) "An improved tone reservation scheme with fast convergence for PAPR reduction in OFDM systems”, IEEE Trans. Broadcast., Vol. 57, No. 4, pp 902-906.

[75] Lv J \& Wan Y, (2013) “An improved tone reservation method for PAPR reduction in OFDM systems", International Conference on Mechatronic Sciences, Electric Engineering and Computer (MEC), Shenyang, China, pp 20-22.

[76] Han D, Yang W \& Liu W, (2014) "A tone reservation scheme based on maximum likelihood method for PAPR reduction in OFDM systems”, High Technology Letters, Vol. 24, No. 4, pp 373-378.

[77] Jiang T, Ni C, Xu C, et al, (2014) "Curve fitting based tone reservation method with low complexity for PAPR reduction in OFDM systems”, IEEE Communications Letters, Vol. 18, No. 5, pp 805-808.

[78] Wattanasuwakull T \& Benjapolakul W, (2005) "PAPR reduction for OFDM transmission by using a method of tone reservation and tone injection", Proc. 5th International Conference on Information, Communications and Signal Processing, Bangkok, Thailand, pp 273-277.

[79] Rahmatallah Y \& Mohan S, (2013) "Peak-to-average power ratio reduction in OFDM systems: A survey and taxonomy”, IEEE Communications surveys \& Tutorials, Vol. 15, No. 4, pp 1567-1592.

[80] Han S H, Cioffi J M \& Lee J H, (2006) "Tone injection with hexagonal constellation for peak-toaverage power ratio reduction in OFDM”, IEEE Commun. Lett., Vol. 10, No. 9, pp 646-648.

[81] Hou J \& Ge J, (2013) "Clipping noise-based tone injection for PAPR reduction in OFDM systems", IEEE International Conference, Budapest, pp 5759-5763.

[82] Jacklin N \& Ding Z, (2013) "A linear programming based tone injection algorithm for PAPR reduction of OFDM and linearly precoded systems”, IEEE Transactions on Circuits and Systems, Vol. 60, No. 7, pp 1937-1945.

[83] Damavandi M G, Abbasfar A \& Michelson D G, (2013) "Peak power reduction of OFDM systems through tone injection via parametric minimum cross-entropy method", IEEE Transactions on Vehicular Technology, Vol. 62, No. 4, pp 1838-1843.

[84] Wang L \& Tellambura C, (2006) "An adaptive-scaling algorithm for OFDM PAR reduction using active constellation extension”, Proc. IEEE Veh. Technology Conf., Montreal, Que, pp 1-5.

[85] Bae K, Andrews J G \& Powers E J, (2010) "Adaptive active constellation extension algorithm for peak-to average ratio reduction in OFDM", IEEE Comm. Lett., Vol. 14, No. 1, pp 39-41.

[86] He J \& Yan Z, (2013) "Improving convergence rate of active constellation extension algorithm for PAPR reduction in OFDM", Proceeding of the IEEE International Conference on Information and Automation, Yinchuan, China, pp 280-284.

[87] Jimenez V P G, Fernandez M S \& Armada A G, (2002) Study and implementation of complementary golay sequences for PAPR reduction in OFDM signals. In: Electrotechnical Conference, MELECON 2002. 11th Mediterranean, Cairo, Egypt, pp 198-203.

[88] Zhang Y, Yongacoglu A, Chouinard J, et al, (1999) "OFDM peak power reduction by sub-blockcoding and its extended versions”, Proc. IEEE Vehicular Technology Conference (VTC), Houston TX, USA, pp 695-699.

[89] Yang K \& Chang S, ( 2003) "Peak-to-average power control in OFDM using standard arrays of linear block codes”, IEEE Commun. Lett., Vol. 7, No. 4, pp 174-176.

[90] Jiang T \& Zhu G, ( 2004) "Complement block coding scheme for reducing peak-to-average power ratio of OFDM systems”, Electronics (China), Vol. 21, No.5, pp 413-420.

[91] Taha Z \& Liu X, (2007) "Low PMEPR code based on STAR-16-QAM constellation for OFDM", IEEE Commun. Lett., Vol. 11, No. 9, pp 747-749.

[92] Liu X \& Wu H, (2010) "Novel asterisk 16QAM constellation for COFDM", IEEE Commun. Lett., Vol. 14, No. 7, pp 596-598.

[93] Huang S, Wu H, Chang S, et al, (2010) "Novel sequence design for low-PMEPR and high-code-rate OFDM systems”, IEEE Trans.Commun., Vol. 58, No. 2, pp 405-410.

[94] Davis J A \& Jedwab J, (1997) "Peak-to-mean power control and error correction for OFDM transmission using golay sequences and reed-muller codes”, IEEE Electronic Letters, Vol. 33, No. 4, pp 267-268. 


\section{AUTHORS}

Zhuo Wang, born in 1991. He is currently working toward the Master degree in Information and Communication Engineering in Beijing University of Technology, Beijing, China. His current research interests include communication and information theory, multicarrier communications and cooperative communications.

Enchang Sun, is a senior member of Chinese Institute of Electronics (CIE), and a chartered member of the Institution of Engineering and Technology (IET) and IEEE senior member. He received the M.Sc. (M.S.E.) degree in Electrical Engineering (EE) from China Academy of Telecommunication Technology (CATT), Beijing, P.R. China, in 2004, and the Ph.D. degree in Information and Telecommunications Engineering from Xidian University, Xi' an, P.R. China, in 2008. Now he is an Associate Professor with the School of Electronic Information and Control Engineering, Beijing University of Technology, Beijing, P.R. China. His current research interests include communication and information theory with special emphasis on wireless digital communications, green communications and networks, and multicarrier communications.

Yanhua Zhang, received the B.E. degree from Xi'an University of Technology, Xi'an, China in 1982, and the M.S. degree from Lanzhou University, Lanzhou, China in 1988. From 1982 to 1990, he was with Jiuquan Satellite Launch Center (JSLC), Jiuquan, China. During the 1990s, he was a visiting Professor at Concordia University, Montreal, Canada. In 1997, he joined Beijing University of Technology, Beijing, China, where he is currently a Professor. His research interests include QoS-aware networking, channel estimation, and radio resource management in wireless communications. He served as the TPC Co-Chair of

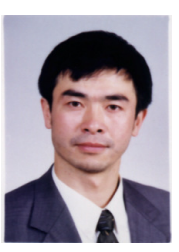
the IEEE ICCCGMCN' 2013, and TPC member of numerous conferences. He has also been a principal investigator of projects of the National High Technical Research and Development Program of China (863 Program), the National Natural Science Foundation of China. 